\title{
La posibilidad combinatoria de nada:una consecuencia para universales inmanentes ${ }^{*}$
}

\author{
Combinatorial Possibility of Nothing: A Consequence for \\ Inmanent Universals
}

\section{Sergio Parra**}

\begin{abstract}
Resumen
Este trabajo se centra en la posibilidad de concebir un Nihilismo Ontológico a partir del combinatorialismo de D. M. Armstrong, según lo han sugerido Efird y Stoneham, aunque presentando una estrategia alternativa al denominado 'argumento de la sustracción'. Según los autores, es posible postular dicho nihilismo mediante las nociones de construcción y estado de cosas de totalidad. Sin embargo, tal hipótesis genera la consecuencia de admitir universales no instanciados en la teoría, es decir, platónicos. Esto último se opone a los requerimientos básicos que fundan la teoría combinatoria, donde ha de imponerse la concepción de universales que únicamente existen en sus instanciaciones. Por ende, asumir una concepción platónica en este ámbito supone un coste muy alto para un naturalismo combinatorio, según el cual sólo existe lo que se encuentra localizado espacio-temporalmente.
\end{abstract}

Palabras clave: combinatorialismo; nihilismo; construcción; estado de cosas de totalidad; universales; D. M. Armstrong.

\footnotetext{
Abstract

This paper focuses on the possibility of conceiving a form of ontological nihilism, starting from D. M. Armstrong's combinatorialism. This possibility has been suggested by Efird and Stoneham, by means of proposing an alternative strategy to the 'subtraction argument'. They claim that it is possible to sustain such nihilism trough the concepts of

* Recibido: marzo 2017. Aceptado: diciembre 2017.

*** Universidad de Valparaíso, Chile. Correo: srparra09@gmail.com
} 
construction and totality state of affairs. However, this hypothesis will require the acceptance of non-instanciated universals, that is, platonic universals. Yet this is opposite to requirements that are basic for a combinatorial theory, which should uphold that universals exist only in their instantiations. Then, the assumption of platonic universals results in high costs for a combinatorial naturalism, according to which only exists what is located in space and time.

Keywords: Combinatorialism; Nihilism; Construction; Totality State of Affairs; Universals; D. M. Armstrong.

\section{Introducción}

Existen varias teorías que intentan dar una explicación ontológica acerca del tipo de entidad que debe ser un mundo posible. En general, las posturas que se pueden destacar suelen pertenecer a dos ámbitos: el Posibilismo Modal y el Actualismo Modal. Por Posibilismo Modal, se entiende un realismo modal extremo que postula que los mundos posibles son entidades de la misma naturaleza que el mundo actual. El Actualismo Modal suele considerarse como un realismo modal más bien moderado, y admite una totalidad de mundos posibles en un espacio ontológico modal constituido por elementos ontológicos del mundo actual. En lo que se refiere al combinatorialismo de Armstrong, éste se cuenta entre las teorías que identifican mundos posibles con combinaciones completas y consistentes de un repertorio de entidades básicas o atómicas. Esta teoría se presenta como un naturalismo actualista, ya que postula que el único mundo concreto es el actual, por lo que sólo existe aquello que se encuentra localizado espacio-temporalmente, y lo que denominamos mundos meramente posibles son construcciones teóricas o abstractas de estados de cosas que representan maneras alternativas de como podrían haber sido las cosas, si no fueran como son de hecho. Por ende, se trata de composiciones no mereológicas de estados de cosas posibles, donde un objeto o $n$-tuplos de objetos pueden, o no, instanciar un universal monádico o poliádico según corresponda a propiedades o relaciones, respectivamente. De esta manera, tenemos conjuntos de estados de cosas de los cuales únicamente existen los estados de cosas actuales. Así, el hecho de que un mundo combinatoriamente posible sea una cierta conjunción de estados de cosas cuyas combinaciones -o recombinaciones-dependan de particulares y universales que existen actualmente, señala una condición bajo la cual no habría lugar para postular hipótesis de corte nihilista, como la posibilidad del mundo vacío. Un mundo es vacío, si no tiene absolutamente ningún objeto concreto. 
Es la posibilidad de que no haya absolutamente nada. Naturalmente, para un realismo naturalista acerca de los mundos posibles, tal posibilidad es difícil de concebir, por lo que corresponde detallar ciertas características relevantes del naturalismo combinatorio de Armstrong.

Considérese, entonces, la noción de independencia como una noción modal, donde un estado de cosas $\mathrm{P}$, por ejemplo, puede darse sin que se dé otro estado de cosas $\mathrm{P}^{*}, \mathrm{y} \mathrm{P}^{*}$ puede darse sin que se dé $\mathrm{P}$. Considérese, además, la noción de mundos contraídos, en virtud de la cual resulta natural pensar en individuos 'contingentes', es decir, que bien podrían no haber existido, por lo que habría mundos con menos objetos que los que hay en el mundo actual. Sin embargo, para Armstrong no es posible llevar a cabo una contracción última que pueda arrojar como resultado la ausencia absoluta de todo lo que existe. Con ello, se sentencia que, si bien no hay nada en particular para lo cual sea necesario existir, el mundo vacío debiera ser pensado como un límite meramente ideal, por tanto, inalcanzable mediante una construcción tal que permita la sustracción gradual de entidades para conjuntos de mundos posibles cada vez más empobrecidos. Así, de la posibilidad de que algo podría no haber existido, no se sigue que podría no haber existido nada en absoluto. Por ello, el más pequeño, el más mínimo mundo posible debe ser, por lo menos, de la forma de un estado de cosas como $\mathrm{F} a$, donde la propiedad $\mathrm{F}$ y el individuo $a$ son simples (Armstrong, 1989, 48).

Es, precisamente, esta condición mínima de la ontología de Armstrong la que es cuestionada por Efird y Stoneham (2006, 269-280), cuyos presupuestos indican que dicha teoría no sólo puede, sino que también debe permitir la posibilidad del mundo vacío. Esto, en el contexto decisivo acerca de cuál metafísica de la modalidad debería preferirse, pues, tal permisividad comportaría la ventaja de proporcionar no sólo una cuenta o explicación acerca de la naturaleza de las posibilidades, sino también sobre qué posibilidades hay. En concreto, esta demanda modal sería: i) intuitivamente plausible, ii) necesaria para quien asuma seriamente la pregunta metafísica tradicional -atribuida a Heidegger (1929) — "¿por qué hay algo más bien que nada?", y iii) se deduce de un principio general e interesante acerca de lo que es posible.

Pese a esto, los autores no advierten que la introducción de un nihilismo en el combinatorialismo de Armstrong, supone costes ontológicos para sus requerimientos en materia de universales. En efecto, los únicos universales que el autor admiten son los denominados universales aristotélicos o inmanentes, a saber, aquellos que sólo existen en una instancia determinada, y su existencia es conocida por investigación empírica, es decir, son necesarios $a$ posteriori, por lo que son descubiertos por la ciencia y no existe para Arm- 
strong absolutamente ningún universal que quede fuera de dicha condición. Además, la posibilidad del mundo vacío, al menos como Efird y Stoneham la proponen, supone un espacio ontológico que no admite relaciones sistemáticas de accesibilidad, puesto que ello, como veremos, se opone a la concepción que el autor tiene con respecto a los universales extranjeros ${ }^{1}$ en el sentido de que son imposibles desde el punto de vista del mundo actual. Por esta misma razón, el desarrollo teórico de las combinaciones nulas depende de ciertos cambios conceptuales, ajenos a las relaciones sistemáticas que se dan en la semántica de mundos posibles y, además, todo lo que pudiésemos dilucidar a partir de dicha suposición se encuentra fuera de los requerimientos que Armstrong hace a los universales, esto es, que estén 'de hecho' instanciados. Así, al asumir la posibilidad del mundo vacío en la teoría combinatoria, sacrificamos dicha condición para dar paso, en el mejor de los casos, a universales que no necesitan estar instanciados para existir, es decir, a una posición platónica con respecto a los mismos.

En la primera parte de este trabajo, se presentará de forma sucinta un bosquejo de la teoría de Armstrong, para luego pasar a una revisión del llamado 'argumento de la sustracción' como fundamento del nihilismo ontológico. Sin embargo, atendiendo a las perspectivas nihilistas dadas para la teoría combinatoria de la posibilidad por Stoneham y Efird, corresponderá revisar los dos ejes desarrollados por los autores para tales efectos, a saber, las nociones de 'construcción' y 'estado de cosas de totalidad'. Finalmente, se advertirá a partir de la concepción que dichos autores tienen de los estados de cosas de totalidad o de segundo orden, cómo es que la teoría debería aceptar universales platónicos.

\section{Teoría Combinatoria de la Posibilidad.}

La ontología de Armstrong admite como constituyentes básicos a particulares, universales y estados de cosas. De esta manera, los individuos instancian universales. La atribución de una propiedad a un individuo es un 'estado de cosas'.

Supóngase que se es un naturalista, sosteniendo que el mundo del espacio-tiempo es todo lo que hay. Supóngase, además, que se postula que este

\footnotetext{
${ }^{1} \mathrm{O}$ universales que, aún siendo posibles, no pueden ser obtenidos de ningún modo a partir del mundo actual. Esta postura frente a esta forma de postular universales Armstrong la mantuvo hasta 1989, pero en 1997 los admite como posibilidades plausibles concebidas en una 'esfera externa' de posibilidad, la cual se encontraría más allá de lo que puede ser obtenido por recombinaciones del repertorio ontológico del mundo actual.
} 
espacio-tiempo tiene una estructura última: una conjunción de estados de cosas cuyos constituyentes son individuos teniendo propiedades y relaciones (universales), y que la identificación de tales universales es un asunto a posteriori. Supóngase, finalmente, que se postula una teoría combinatoria de la posibilidad, sosteniendo, en particular, que todas las meras posibilidades son recombinaciones (no-existentes) de elementos actuales (Armstrong, 1989: $54)$.

Esto se enmarca en una posición que pretende evitar compromisos ontológicos con estados de cosas negativos, pues supondría también que hay universales negativos. Por otro lado, lo que aquí tiene lugar es la posibilidad de variación independiente de un repertorio de entidades atómicas las cuales, en razón de su independencia mutua, pueden darse o no darse en un determinado mundo posible, sin que esto tenga mayor implicación con el hecho de que se den o no los demás estados de cosas. Ahora bien, también es cierto que, según esto, no hay más estados de cosas que se configuren 'fuera' de los estados de cosas actuales, por lo que los estados de cosas posibles dependen de la recombinación independiente de los individuos e universales que de hecho hay. Esta manera de reconstruir mundos posibles es lo que puede considerarse una 'estrategia ficcionalista' ${ }^{2}$, donde los estados de cosas posibles no tienen existencia concreta. Por ende, cabe preguntarse de qué manera son elaboradas tales construcciones ficcionales, lo cual, a fin de cuentas, depende de la ontología con la cual operemos.

Primero, consideraremos un número no especificado de particulares de primer orden - sean, por ejemplo, individuos como $a, b, c$, etc.-, los cuales son simples en el sentido de que no poseen como partes propias a otros individuos. Considérese, además, un número indeterminado de propiedades también simples, sean, por ejemplo, F, G, H, etc. Estas serán universales, los cuales, a su vez, pueden ser divididos en relaciones para los casos de instan-

\footnotetext{
${ }^{2}$ Nótese que, en este sentido, si los mundos posibles son considerados ficciones, entonces, tal como Armstrong apunta, no hay mayores problemas para admitir la inteligibilidad de mundos imposibles, o bien, mundos doxásticamente posibles en cuanto ficciones también. Por ejemplo, si consideramos la proposición acerca de si "la serie de pares primos es infinita" tenemos, en términos de concebibilidad que, simultáneamente, es concebible que esto sea cierto, así como que sea falso, pues, en rigor, no es posible un procedimiento de verificación por casos para determinar el valor de verdad de proposiciones que versan acerca de series infinitas. Esto, entonces, no sería susceptible de ser tratado en términos realistas, pero sí en el ámbito de un ficcionalismo en orden al cual puedan considerarse entidades imposibles como instrumentos-de-pensamiento útiles para esclarecer problemas sobre actualismo.
} 
ciación poliádica ${ }^{3}$ y propiedades para los casos de instanciación monádica ${ }^{4}$. Dado esto, vamos a suponer un caso de simplicidad extrema donde los estados de cosas actuales podrían agotarse en el siguiente ejemplo:

\section{I) $\mathrm{F} a \& \mathrm{G} b \& \mathrm{H} c$}

Ahora, lo primero que un combinatorialista podrá pensar, es que dichos estados de cosas actuales podrán recombinarse de las siguientes formas:

II) $\mathrm{F} b \& \mathrm{G} a \& \mathrm{H} c$

III) $\mathrm{F} c \& \mathrm{G} a \& \mathrm{H} b$

En términos explicativos, basta considerar estas dos posibilidades de variación, las cuales serían mundos posibles o conjuntos de posibles estados de cosas. Lo que no puede considerarse, estará reservado para estados de cosas posibles disjuntos y negativos, donde, por un lado, siendo $\mathrm{F}$ y $\mathrm{G}$ propiedades, éstas no pueden constituir la propiedad 'ser F o G', así, de esta forma. Por otro lado, tampoco es admisible la propiedad de 'no ser F' o 'no ser G', pues, en efecto, que $a$ sea $\mathrm{F}$, tiene que ver con la situación predicativa donde se señala que $a$ no es G, pero no se trata de la propiedad 'no ser G', sino, más bien, del estado de cosas positivo de ser $a$ un F, nada más. En este sentido, existe un primer rechazo categórico de los estados de cosas negativos, por lo que 'no ser algo' no puede constituir un genuino estado de cosas, además del hecho de que podemos aceptar que diferentes mundos varíen respecto a sus dominios de objetos y universales, o sea, que bien puede darse el caso de objetos que existen en el mundo actual, pero que se encuentran ausentes en otros mundos posibles que, por lo demás, no son un conjunto de mundos independientes entre sí, sino un conjunto de mundos que son posibles unos respecto de otros. En otras palabras, se trata de posibilidades relativas $R$, que implican relaciones de accesibilidad reflexivas y transitivas ${ }^{5}$. De esta manera, el mundo actual puede estar constituido por los estados de cosas que aparecen en el ejemplo (I) y, asimismo, puede haber un estado de cosas posible directamente accesible al estado de cosas actual, constituido por los estados de cosas $\mathrm{F} b$ \& $\mathrm{G} a$, donde podemos notar que $\mathrm{H} c$ no aparece. Si avanzamos, por tanto, en la contracción, podemos obtener un mundo posible constituido por el único estado de cosas $\mathrm{F} a$, pero no es posible para Armstrong ir más allá de eso, puesto

\footnotetext{
${ }^{3}$ Como que un grupo de personas se encuentre en una habitación, por ejemplo, donde 'encontrarse en una habitación' sería el universal poliádico, es decir, que se instancia en más de un caso.

${ }^{4}$ En el caso de un individuo, puede ser el tener cierto peso, masa, altura, etc.

${ }^{5}$ En este aspecto, seguimos la noción de "estructura modelo" en Kripke. Véase Nubiola, Jaime. El compromiso esencialista de la Lógica Modal. Eunsa, Pamplona, 1991, p. 153 y ss.
} 
que, aquello que es posible depende, para su construcción, de los elementos del mundo actual, por lo que se vuelve necesario que al menos haya algo, ya que "el más pequeño mundo posible será un estado de cosas de la forma $\mathrm{F} a$, donde F y $a$ son simples" (Armstrong, 1989, 64) ${ }^{6}$, a lo cual sumamos la consideración de que todo particular debe instanciar al menos un universal, y todo universal debe estar instanciado en por lo menos un particular. Así, en la serie de mundos cada vez más contraídos la posibilidad del mundo vacío señala un límite ideal y, como sucede con ciertos límites, éste no puede ser alcanzado. No obstante, existen posiciones argumentativas favorables a tal posibilidad, donde si es posible plantear un estado de cosas tal.

\section{El Argumento de la Sustracción y lo concreto}

Ante todo, permítasenos comenzar diciendo que aquello que se denomina 'nihilismo metafísico' comenzó a ser tomado con seriedad a partir de los trabajos que en 1996 los filósofos Peter van Invagen y Jonathan Lowe presentaron en la Joint Session de Dublin (Van Inwagen, 1996, 95-110; Lowe, 1996, 111-120), siendo Lowe el responsable de acuñar el término en cuestión. Sin embargo, sería Thomas Baldwin (1996: 231-238) quien establecería una defensa plausible a partir de lo que se entiende como el argumento de la sustracción. El argumento discurre de la siguiente forma:

(A1) Podría haber habido un número finito de objetos concretos.

(A2) Cada uno de aquellos objetos concretos es contingente.

(A3) La no-existencia de cualquiera de aquellas cosas no haría necesaria la existencia de cualquier otra.

Evidentemente, no sabemos cuántos son los objetos que existen, pues éstos podrían ser infinitos, por lo cual, en (A1) se presupone un número finito para lo que existe, por lo que esto nos permite situar un punto de inicio para llevar a cabo la contracción. Siguiéndose de lo anterior, (A2) nos dice que cualquier objeto en ese mundo podría no haber existido. Como señalara Coggins $(2008,229-237)$ habría, en primer término, un mundo $w_{1}$ que, entre otras cosas, contiene al objeto $x_{1}$. Pero, relativamente a $w_{1}$ hay un mundo $w_{2}$ que contiene todos los objetos que se encuentran en $w_{1}$, excepto $x_{1}$ Por ende, a la inversa, $w_{2}$ no contiene nada que no exista ya en $w_{1}$ que es lo que (A3) dice al afirmar que ninguna existencia particular va a implicar por necesidad la

\footnotetext{
${ }^{6}$ Asimismo, entiéndase por expansión, el caso para cuyos mundos que envuelven más individuos que aquellos que existen actualmente; y, por contracción, cuando los mundos en cuestión envuelven un subconjunto propio de individuos e universales actuales.
} 
existencia de otra. Por ende, podemos continuar haciendo la reducción hasta alcanzar un mundo denominado $w_{\min } \cdot w_{\min }$ que contiene un objeto o grupo de objetos tales que, si uno de ellos no existiese, entonces ninguno existiría. Esto nos permite dar el paso a un mundo $w_{\text {nil }} \cdot w_{\text {nil }}$ donde no hay objetos concretos. Por lo tanto, bien podría no haber habido nada.

Consiguientemente, este argumento ha sido modificado y reforzado en muchas versiones, pero aquello que aquí es relevante es que la existencia de los objetos concretos responde a una contingencia tal, que nos puede llevar a la formulación de la contingencia de absolutamente todo lo que existe. Sin embargo, el argumento aún puede descansar sobre premisas suficientemente débiles como para descartarlo. Así, Rodriguez-Pereyra (1997, 159-166) observa que (A1) resulta poco convincente, pues parte de la suposición de que el total de objetos concretos es finito, donde concreto se refiere a tener partes espacio-temporales. Así, bien puede pensarse que, necesariamente, si algo tiene alguna parte espacio-temporal, entonces tiene muchas de forma indefinida. Además, necesariamente las partes de un objeto concreto son objetos concretos también. Luego, es necesario que los objetos concretos tengan una infinitud de partes concretas. Por otro lado, también se puede pensar que, siendo necesario que si algo existe, entonces existen conjuntos que lo contienen como un $u r$-elemento ${ }^{7}$, y que por necesidad los conjuntos cuyos $u r$-elementos son concretos, son ellos mismo concretos también. Si esto es así, entonces es cierto que no existe un mundo posible que tenga algún objeto concreto $\mathrm{y}$, de manera finita, muchos objetos tales. Con ello, se observa que existe un problema definicional con aquello que entendemos como 'concreto', por lo que Rodriguez-Pereyra desarrolla la noción de objeto concreto* como aquel que es parte, así como un ocupante máximo de una región determinada. Así entonces, tenemos que:

(i) Podría haber un número finito de objetos concretos*

(ii) Si hay un mundo sin objetos concretos*, entonces ese mundo no tiene objetos concretos en absoluto.

Con ello (A1) descansará sobre (i) y (ii), sin correr el riesgo de invalidar el argumento, al menos en el sentido explicado.

Por otro lado, es preciso acentuar que, según esta definición, un objeto concreto* debe ocupar máximamente una región espacial, lo cual es una manera de establecer que éste y sólo este objeto puede ocupar dicha región, a lo que añadiremos, junto a Efird y Stoneham, en un determinado momento o

\footnotetext{
${ }^{7}$ Un $u r$-elemento es un objeto que pertenece a un conjunto, sin ser él mismo un conjunto.
} 
instante: "Un objeto concreto es aquel que existe en alguna locación del espacio-tiempo, tiene alguna cualidad intrínseca, y es tal que si éste tiene un límite, entonces se trata de un límite natural" (Efird y Stoneham, 2009, 132-137). Con ello, la determinación de los objetos que poseen una ubicación espacio temporal queda resguardada, por lo que, hasta el momento, todo lo que se ha dicho de los objetos, incluso en versiones más elaboradas del argumento de la sustracción, no entra en conflicto directo con el naturalismo actualista de Armstrong, salvo por la conclusión del argumento.

\section{Las nociones de construcción" y totalidad de estados de cosas}

En lo que sigue, centraré mi atención en dos aspectos que, a mi parecer, son decisivos para dar cumplimiento a los objetivos teóricos que propone la hipótesis de combinación nula de Efird y Stoneham. En primer lugar, intentaré dilucidar el sentido del término 'construcción', según lo dicho por los autores. Ciertamente, ellos se permiten una alternativa metodológica para ampliar el sentido restrictivo acerca de lo que es combinatoriamente posible, motivados, en parte, por la noción de 'conjunción única' que tiene lugar en Armstrong, puesto que el filósofo australiano no piensa un mundo solamente como una conjunción de dos o más estados de cosas juntos, sino que, en un sentido más bien extendido del término conjunción, se admitirá que los mundos posibles pueden consistir en la conjunción de sólo un estado de cosas. La autorización para este procedimiento vendría dada por la admisión de posibilidades simples, es decir, para la forma última de un individuo como $\mathrm{F} a$, lo cual podría significar, por ejemplo, que hay un mundo con tan sólo un objeto ejemplificando una determinada propiedad.

Ello debiera llevarnos al segundo punto de importancia, es decir, a la consideración unitaria de la totalidad de los estados de cosas: el estado de cosas de totalidad. Nótese que no es igual referirse a (i) la totalidad de los estados de cosas, que a (ii) el estado de cosas de totalidad. Mientras que (i) se refiere a todos los estados de cosas de orden inferior o primer orden, (ii) hace referencia al estado de cosas de orden superior que consiste en la existencia de todos los estados de cosas de orden inferior. En otras palabras, todos los estados de cosas constituyendo un estado de cosas.

Ahora bien, en primer lugar, el que los estados de cosas posibles sean construidos hace referencia a la operación lógica de la conjunción. Así, los mundos posibles son conjunciones de estados de cosas que incluyen a la conjunción única. Dado esto, habría más de una manera en que el término construcción —como metáfora para el caso de las posibilidades- podría ser 
desarrollado de tal manera que permita, por un lado, el mundo vacío, y otra en la que no. Para este caso, aún nos encontramos en una posición neutral que no nos fuerza a tomar partido por una u otra forma. Así entendemos, entonces, el primer sentido de construcción como un proceso en el cual siempre se tiene un determinado producto, lo cual es compatible con la definición combinatoria dada en la teoría de Armstrong. En segundo lugar, defínase una noción de construcción* sobre un conjunto de elementos que permitan el producto vacío o nulo. Por ejemplo, supongamos que contamos con un conjunto de elementos dados en una caja y que, al mismo tiempo, contamos con una superficie en blanco que, para el caso, no representa o no ilustra nada en sí misma. En primera instancia, para un combinatorialista como Armstrong, el que la superficie no represente nada es condición para descartar la posibilidad del mundo vacío, por lo que las únicas posibilidades que arrojen un resultado concreto dependerán de que los elementos o ítems de la caja sean incluidos y dispuestos de diferente forma en dicha superficie. Así, diferentes disposiciones son diferentes mundos posibles. Frente a esto, Efird y Stoneham reclaman que, si la analogía del tablero o superficie es determinante para visualizar qué es parte, o no, de la representación de un mundo posible, bien puede llevarse a cabo un ejercicio similar donde no sólo es válido dejar en la caja los elementos no seleccionados, sino también el que nos propongamos la tarea de representar un mundo posible dejando absolutamente todos los elementos dados en la caja, sin incluirlos en el lienzo, para así señalar nuestra representación apuntando prácticamente a nada. Luego, los elementos del mundo actual han sido usados para representar el mundo vacío. Nótese, además, que el término 'representación' alude al hecho de que el combinatorialista usa los elementos espacio-temporales para la representación de posibilidades no actuales, por lo que se trataría de un ejercicio de tipo creativo. Después de todo, para Armstrong, sólo podemos referirnos a los mundos posibles en términos de apariencia y ficción (Armstrong, 1989, 49).

Volviendo sobre lo anterior, los autores enfatizan que aquello que solemos entender naturalmente como conjunción, involucra la reunión de dos o más objetos cualesquiera, pero Armstrong extiende la comprensión de esta noción al admitir que los mundos posibles puedan consistir en la conjunción unitaria de un estado de cosas, en orden a cobijar las posibilidades más simples ${ }^{8}$. Por ejemplo, un mundo está constituido por un conjunto de estados de cosas. Ahora, la forma de especificación más simple para un mundo posible está en decir que se trata de cualquier conjunción de posibles estados de cosas ató-

\footnotetext{
${ }^{8}$ Es decir, haciendo la extensión definicional de conjunción unitaria, me permito tener por lo menos un objeto.
} 
micos, a los cuales se añade la conjunción unitaria de todos estos estados de cosas. Notemos que la noción de conjunción unitaria o única es una extensión para lo que se entiende como conjunción. La noción común de conjunción, como ya sabemos, es el resultado de juntar dos o más cosas, es decir, tiene que haber más de un conyunto. La noción propuesta por Armstrong admite que los mundos posibles consistan en la conjunción unitaria de estados de cosas, en primer lugar, porque esta amplitud definicional es compatible con la noción de mundos contraídos. El mundo más contraído posible ha de admitir a lo menos un estado de cosas de la forma $\mathrm{F} a$, como se dijo. En segundo lugar, todo individual debe estar instanciando a lo menos una propiedad. No hay individuos 'desnudos' desprovistos de toda propiedad. En una serie de individuos de este tipo la única diferencia que puede establecerse entre uno y otro es meramente numérica, lo que es el caso para: $x_{1}, x_{2}, x_{3}, \ldots, x_{\mathrm{n}}$, que no es otra cosa que un ejercicio teórico de abstracción; pero en los hechos, o incluso en los estados de cosas posibles donde hay objetos, todo individuo, sea $a$, debe instanciar al menos una propiedad para calificar como estado de cosas mínimo.

Ahora, si se admite esta extensión definicional para la noción de conjunción, no se explicaría la resistencia de Armstrong para aceptar las conjunciones nulas o vacías, las cuales están bien-definidas en la teoría lógica de las formas normales donde una conjunción nula aparece como trivialmente cierta. Así, la estrategia de ambos autores tiene mayor relación con una dimensión de análisis conceptual, que con demandas sistemáticas en términos estrictamente modales, por lo que debiera admitirse, con Armstrong, que no hay relaciones de accesibilidad con tales mundos. Esta falta de accesibilidad se explica por las dificultades de Armstrong para aceptar los universales 'extranjeros' postulados por D. K. Lewis $(1986,91)$. Según este autor, una propiedad es extranjera a un mundo si y sólo si no está instanciada por ningún objeto de ese mundo, y si no es definida o analizable como una propiedad conjuntiva o estructural constituída por las propiedades instanciadas en los objetos de ese mundo. Asimismo, un individuo que instancie propiedades de este tipo, ha de ser extranjero también. Para comprender mejor esta idea, Armstrong nos invita a pensar en la perspectiva de que nuestro mundo sea extranjero con respecto a un mundo más empobrecido que el nuestro en términos de constituyentes. Concentrándonos en el caso de las propiedades, podemos decir que muchas propiedades de este, nuestro mundo, son extranjeras en relación a esos mundos con un repertorio ontológico menor. Considérese al mundo actual como $\mathrm{W}_{a}$, y al mundo más pequeño o contraído con respecto a este lo designaremos por $\mathrm{W}_{\mathrm{c}}$. Diremos así que $\mathrm{W}_{a}$ contiene individuos ins- 
tanciando la propiedad $\mathrm{F}$, pero no hay ningún individuo instanciando dicha propiedad en $\mathrm{W}_{\mathrm{c}}$, o bien, simplemente dicha propiedad no existe en $\mathrm{W}_{\mathrm{c}}$. De ello se sigue, por relaciones de accesibilidad, que $\mathrm{W}_{c}$ es accesible desde $\mathrm{W}_{a}, \mathrm{o}$ que es un mundo posible relativo a $\mathrm{W}_{a}$. Pero, si $\mathrm{F}$ es una propiedad extranjera respecto de $\mathrm{W}_{c}$, entonces no la podemos obtener ni por la conjunción ni por la recombinación de ninguna de las propiedades instanciadas en $\mathrm{W}_{\mathrm{c}}$. De esta manera, $\mathrm{W}_{a}$ no es accesible desde $\mathrm{W}_{\mathrm{c}}$. Si en relación a $\mathrm{W}_{\mathrm{c}}$ la propiedad $\mathrm{F}$ es extranjera, entonces la simetría falla9. La importancia que esto último tiene para la accesibilidad a un mundo vacío es que éste, al no tener propiedad alguna, admite un punto de vista en orden al cual todo mundo con al menos una propiedad es extranjero respecto a dicho mundo vacío. Ningún mundo es accesible a partir del mundo vacío, porque todo mundo sería extranjero para un mundo que no contiene nada.

Pasamos al segundo aspecto de interés. Se trata de los estados de cosas de totalidad, los cuales son introducidos por Armstrong para proporcionar 'verificadores ${ }^{10}$ para afirmaciones acerca de estados de cosas y universales negativos. Así, los autores señalan que tal condición se introduce mediante una relación de totalización entre estados de cosas de primer orden, por lo cual, tanto el mundo actual, así como cada mundo posible deben requerir al menos un estado de cosas de segundo orden. Ya en la introducción de su teoría combinatoria de la posibilidad, Armstrong admite que el mundo no consta únicamente de estados de cosas de primer orden, por lo que se requiere, a lo menos, de un estado de cosas de orden superior o de totalidad, tal que sea un hecho (general) acerca de la conjunción de todos los estados de cosas de primer orden. Lo que se pone de relieve en el trabajo de Efird y Stoneham, es que la concepción de estados de cosas de totalidad, bien podría adecuarse a una concepción del mundo vacío, puesto que, siendo contingentes todos los estados de cosas de primer orden, este mundo es precisado como "el estado de cosas de segundo orden que consiste en no haber estados de cosas de primer orden" (Efird y Stoneham, 2006, 278), pues, si resulta admisible el paso conceptual para entender la conjunción de los estados de cosas como unitariedad, nada impediría concebir un estado de cosas de orden superior que no contenga absolutamente ningún estado de cosas de orden inferior, o sea, una conjunción nula.

\footnotetext{
${ }^{9}$ Pues la accesibilidad no se cumple en ambos sentidos para los dos mundos.

${ }^{10}$ Traducción tentativa para la expresión inglesa 'truthmaker', es decir, algo en el mundo que hace verdadera a una proposición. Otras traducciones asumen el término "Hacedor de Verdad", pero muchos autores consideran que "verificador" cumple con los requerimientos de la definición de 'truthmaker'.
} 
Sin restar mérito a lo expresado hasta aquí por ambos autores, me detendré en la caracterización de los estados de cosas de totalidad como hechos de clausura, es decir, como límites en virtud de los cuales es cierto que no hay nada más aparte de tales límites. De esta manera, podemos referirnos a la totalidad de los estados de cosas del mundo como 'el estado de cosas de totalidad T'. Como ha señalado Pagès ( 2001, 63-84), esta totalidad, en tanto es la única que hay, es una verdad que puede expresarse mediante un existencial negativo, a saber, 'no existe ningún estado de cosas distinto de los que constituyen la totalidad T'. Armstrong entiende que si es verdad que cierta conjunción de estados de cosas es la totalidad de estados de cosas, esto es verdadero sólo porque no hay más estados de cosas, ya que, de haber más, entonces la proposición simplemente no es cierta. Así, 'que no haya más estados de cosas' ha de ser, de alguna manera, agregado al verificador, el cual, a su vez, debe ser el estado de cosas consistente en la gran conjunción de todos los estados de cosas. Pareciera ser, entonces, que la totalidad de estados de cosas, por cuanto supone un límite, cumple con las mismas características que el propio Armstrong señala para los límites (Armstrong, 2004, 53), esto es, que son verdades negativas en tanto no hay nada más allá de dicho límite absoluto. Ahora, Efird y Stoneham están conscientes de que verdades como 'esto es todo lo que hay' y 'no hay nada más' son, en el fondo, dos caras de la misma moneda. Una, concebida como una acepción positiva de la totalidad existencial y, la otra, como la negación implícita que ésta conlleva. El razonamiento para una conclusión tal parece bastante obvio, pues, si un estado de cosas cualquiera es contingente, entonces, la totalidad de estados de cosas concebida 'unitariamente' como 'el estado de cosas de totalidad' ha de ser contingente también, por lo que la actual existencia de todo traería aparejada, por necesidad, a su correlato antagónico, a saber, la posibilidad de nada.

\section{Sustracción para universales: más allá de un naturalismo combinato- rio}

Según el curso de las anteriores afirmaciones, entonces, el paso conceptual dado por los autores permite suponer un estado de cosas de segundo orden sin estados de cosas de primer orden. Pero, al modo de ver de Armstrong, los estados de cosas de orden superior son sólo necesarios porque presuponen la existencia de los estados de cosas de orden inferior. Si bien esto puede parecer gratuito, ha de ilustrarse tomando en cuenta que ciertas propiedades como ser un kilo, ser un cuarto o una media tonelada, por ejemplo, caen, a su vez, bajo el universal ser masa. Con ello se dice que K (ser un kilo), cae bajo $\mathrm{M}$ (ser masa), donde $\mathrm{M}(\mathrm{K})$ es un estado de cosas de segundo orden, por lo 
cual, si tenemos que $a$ cae bajo $\mathrm{K}$ y K cae bajo M, entonces, $a$ cae bajo M. Esto constituye una necesidad en el sentido en el que reconocemos que no podría ser que $a$ sea $\mathrm{K}$ sin ser M, es decir, que pueda pesar un kilo sin tener masa. Visto desde esta perspectiva M (K) depende del primer orden en el que $a$ tiene lugar. Con ello, debiera admitirse, entonces, que es difícil pensar estados de cosas de orden superior que existan sin sus constituyentes, es decir, sin los estados de cosas de orden inferior. Pero, como estamos pensando la posibilidad de nada, nos hemos de referir al estado de cosas de segundo orden por excelencia, a aquella totalidad $\mathrm{T}$, la cual queda establecida como hecho de clausura para todo lo que hay. Pero, al dar este paso, ¿qué sucedería con los demás universales?

\subsection{Universales no instanciados}

Existen consideraciones que nos proveen de una versión de la sustracción para universales ${ }^{11}$. Estas se basan en dos premisas básicas:

i) La contingencia de las diferentes instanciaciones de un universal.

ii) La independencia ontológica entre estas instanciaciones.

La primera se refiere a la posibilidad de postular mundos metafísicamente posibles en donde las instanciaciones de un universal son sustraídas. Luego, habríamos de llegar a un mundo posible donde un solo individuo instancia al universal. La segunda, se acerca al principio de independencia del que ya se ha hablado aquí y el cual establece que un estado de cosas P puede darse sin que se dé otro estado de cosas $\mathrm{P}^{*}$, y $\mathrm{P}^{*}$ puede darse sin que se dé P. Pero, interpretado de una manera particular, dicho principio nos dirá aquí que dichas instanciaciones pueden ser sustraídas hasta postular un mundo posible en donde ningún objeto instancia a un determinado universal. Es decir, se puede postular la posibilidad de universales a priori, que no dependen de determinaciones empíricas para existir y que, por ello, no requieren estar instanciados en ningún objeto. Esto es diametralmente opuesto a la defensa que Armstrong hace de los universales inmanentes, a saber, aquellos que únicamente pueden existir en sus instancias. Se supone así, que para Armstrong no habría más que estos universales, los cuales son descubiertos en el marco del ejercicio científico, por determinaciones empíricas, de manera a posteriori.

\footnotetext{
${ }^{11}$ Para estas precisiones me basaré en Alvarado, J. T. "El argumento de sustracción para universales trascendentes”, en KRITERION, n 125, 2012, p. 263-269.
} 
Notemos, según lo anterior, que la sustracción para universales no instanciados es compatible con la propuesta de Efird y Stoneham, ya que, si para estos últimos los estados de cosas de primer orden son contingentes, esto sólo puede sostenerse bajo la condición de mantener los estados de cosas de orden superior en un ámbito de autosuficiencia, es decir, no tendrían por qué ser dependientes de un primer orden, conservando así un status ontológico propio. Si esto es así, tenemos que acceder a la comprensión de la contingencia de todo lo que hay sólo a través de los estados de cosas de orden superior, pues, el argumento exige que haya, por lo menos, un estado de cosas de este tipo para sostener dicha accesibilidad conceptual. Así, lo que se denomina platonismo en materia de universales sostiene la posibilidad de la existencia de universales cuya naturaleza es tal, que podría no estar instanciado por nada. Por lo demás, ya hemos visto que el ámbito de un segundo orden en Armstrong comprende relaciones y propiedades de universales. Por lo tanto, quien defienda el carácter autosuficiente de la existencia de dicho ámbito bien puede comprometerse con un platonismo, el cual niega la tesis inmanentista según la cual todos los universales deben encontrarse esencialmente instanciados en algún individuo.

Pese a esto, aún se podría reprochar que hay una diferencia fundamental -en los términos de un nihilismo ontológico- al referirnos al ámbito del estado de cosas de totalidad y al de los universales, por cuanto estos últimos caen bajo dicha totalidad, en cuyo caso la contingencia de la instanciación de cualquier universal no implica por necesidad la contingencia de la existencia de los individuos, puesto que, si admitimos la sustracción gradual de las instanciaciones de un universal hasta que quede un solo individuo instanciando dicha propiedad, para luego postular mundos posibles donde ningún particular instancie el universal; de esto no se sigue que absolutamente todos los universales sean no instanciados y que, por lo tanto, pueda no haber individuos en absoluto. Ciertamente, creo que sí podríamos pensar mundos metafísicamente posibles donde sólo haya universales no instanciados, aunque con ciertas precisiones que el defensor de un platonismo podría establecer, pero eso es diferente a la afirmación sobre la posibilidad de que no haya absolutamente nada.

\section{Conclusiones}

Hemos visto que el argumento de la sustracción en su versión más reforzada parece no ser del todo incompatible con el combinatorialismo de Armstrong, por lo menos en lo que se refiere a la cualificación de objetos con- 
cretos. Aunque debiera admitirse la salvedad de la existencia de a lo menos un individuo para que pueda constituirse un mundo posible en los términos del combinatorialismo, lo cual nos ha llevado a las formulaciones hechas por Efird y Stoneham sobre la posibilidad combinatoria del mundo vacío. Pero si hemos de dar crédito a tales argumentos, debemos admitir los costes ontológicos que éstos traen aparejados. En efecto, la noción de un estado de cosas de segundo orden que consiste en que no haya estados de cosas de primer orden, supone la independencia ontológica de dicho orden superior que es, por lo demás, donde tienen lugar los universales y las relaciones entre éstos. Si esto es así, no habría mayores razones para adquirir compromisos fuertes con los universales aristotélicos que Armstrong postula, pero con ello se sacrifica la formulación de un naturalismo combinatorio para dar cuenta acerca de la naturaleza de los mundos posibles.

Finalmente, pareciera ser que existe una relación estrecha, aunque problemática, entre lo concreto actual y la nada, sobre todo a la hora de postular la gran totalidad de estados de cosas, por lo cual, el supuesto de amplitud conceptual de las posibilidades que proponen Efird y Stoneham ya estaría implícito, de alguna manera, en el hecho de que la noción de límite ya incluiría una dimensión ontológica aún no bien definida. Por lo mismo, habría que reconocer que tales problemas para la teoría de Armstrong motivan a desarrollos ulteriores que bien pueden comportar las ganancias y pérdidas que deben asumirse en determinadas elecciones teóricas.

\section{Referencias}

Alvarado, J. T. (2012). "El argumento de sustracción para universales trascendentes". En KRITERION, № 125.

Armstrong, David M. (1989). A Combinatorial Theory of Possibility. Cambridge: Cambridge University Press.

Armstrong, D. M. (1997). A World of States of Affairs. Cambridge: Cambridge University Press.

Armstrong, D. M. (2004). Truth and Truthmakers. Cambridge: Cambridge University Press.

Baldwin, Tom (1996). "There might be nothing". En Analysis, № 56.

Coggins, Geraldine (2008). "Metaphysical Nihilism". En Philosophical Books, No 3, Vol. 49, pp. 229-237. 
Effird, D. \& Stoneham, T. (2006). "Combinatorialism and The Possibility of Nothing". En Australasian Journal of Philosophy, N 84, Vol. 2.

Effird, D. \& Stoneham, T. (2009). "Justifying Metaphysical Nihilism: A response to Cameron". En The Philosophical Quarterly, N²34, Vol. 59.

Heidegger, M. (1967). ¿Qué es metafísica? Buenos Aires: Siglo Veinte.

Lewis, D. K. (1986). On the Plurality of Worlds. Oxford: Blackwell.

Nubiola, Jaime (1991). El compromiso esencialista de la Lógica Modal. Pamplona: Eunsa.

Pagés, Joan (2001). "Identidad de tipos y hacedores de verdad". En Crítica: Revista Hispanoamericana de Filosofía.

Rodriguez-Peryra, Gonzalo (1997). "There Might Be Nothing: The Subtraction Argument Improved". En Analysis, № 57.

Van Inwagen, Peter (1996). "Why Is There Anything At All?: I". En Aristotelian Society, Supplementary Volume 70.

Lowe, E.J. (1996). "Why Is There Anything At All?: II". En Aristotelian Society, Supplementary Volume 70. 
